\title{
Establishment of Supply And Demand Match Indicators for Taxi Resource \\ Yilin Song ${ }^{1}$
}

${ }^{1}$ School of Energy, Power and Mechanical Engineering, North China Electric Power University, Beijing 102206, China

Keywords: Internet + , cluster analysis, multi-objective programming

\begin{abstract}
With the coming of "Internet +" era, drops a taxi, Uber and other companies rely on mobile Internet software to establish a taxi service platform, and achieve information exchange between the passengers and the drivers. Aiming at this phenomenon, based on in the history data in a certain period at designated observation point of Beijing to analyze the taxi resources match and the difficulty of taking a taxi. From the sky drops and quick smart travel platform, this paper extracts 11 sample locations in Beijing such as Jianguomen, Fuxingmen of taxi distribution and demand in 22 sample time from September 6th to May 7th, 2015, taking the number of people using the drops one million and drops penetration in the taxi industry, to estimate Beijing's taxi operations (supply and demand). In order to establish a reasonable target, we should analyze the match level of supply and demand of different space-time taxi resource. To establish the related matching degree of the taxi demand supply indicators (defined as the difference rate), this paper uses control variables to analyze taxi resource matching degree of sample locations and sample time in Beijing. Use cluster analysis method to get the conclusion of 11 observation points: The matching degree in Fuxingmen, Xizhimen and Zhongguancun is low, and that of Jianguomen, Dongzhimen and Xisanqi is higher. The matching degree of weekend daytime and weekday evening peak (7:00-10:00, 17:00-20: 00) is lower compared with other periods.
\end{abstract}

\section{Problem analysis}

First, by analyzing the influence of taxi distribution amount and taking taxi demand on the matching degree of taxi resources, the matching degree indicators of supply and demand evaluation of taxi resource are established. From the sky drops fast smart travel platform, this article extract the taxi distribution and demand condition in Beijing. The variable control method is adopted to analyze the supply and demand matching degree of different space taxi resource and the matching index is calculated based on statistical data. Finally, cluster analysis method is used to evaluate the matching degree of taxi resources at different time.

\section{Symbol conventions}

\begin{tabular}{|c|c|}
\hline Symbol & Meaning of symbols \\
\hline$m_{i j}$ & The taxi supply amount in the j-th place at the i-th time \\
\hline$n_{i j}$ & The taking a taxi demand amount in the j-th place at the i-th time \\
\hline$p_{i j}$ & The supply and demand matching degree index in the j-th place at the i-th time \\
\hline$l$ & Mileage utilization \\
\hline$w$ & The incentive amount per hour \\
\hline
\end{tabular}




\section{Assumptions of the model}

(1) Ignore the influence of uncertain and irresistible factors such as bad weather, traffic jams, traffic accidents and others on rally car.

(2) The effect of black car phenomenon on the reliability of the collected data is negligible.

(3) Within a period of time, considering the supply of taxis within the region, the greater the success possibility of a passenger taking a car is, the more easily to take a taxi.

\section{Establishment and solution of the model}

Establishment of model evaluation index.Taxi supply refers to the number of taxis which are available for services within $2 \mathrm{~km}$ range near a particular location at a certain time. Taxi demand refers to the number of taxi orders in a particular place at a certain time.

As the two most important factors that affect the supply and demand matching degree of taxi resources, the taxi distribution and taking a taxi demand directly relate to taxi resource supply and demand, which is an important part of the evaluation. Therefore, in order to evaluate the supply and demand match level in particular location at a certain time, we define difference rate $\mathrm{p}$ as an index for match evaluation, which is calculated as follows:

$$
p_{i j}=\frac{\left|n_{i j}-m_{i j}\right|}{n_{i j}}
$$

Where, $n_{\mathrm{ij}}$ and $m_{\mathrm{ij}}$ denote the taxi demand and supply in the $\mathrm{j}$-th place at the $\mathrm{i}$-th time. Difference rate $\mathrm{p}$ is a number greater than 0 , the closer $\mathrm{p}$ value is to 0 , the higher it indicates the matching degree of supply and demand. The bigger difference rate $\mathrm{p}$ is, the lower it indicates the matching degree of supply and demand, and the worse the effect is.

\section{Use cluster analysis to evaluate the matching degree of supply and demand}

\section{Model analysis}

The basic principles of cluster analysis of samples are: Firstly, regard a certain number of sample as a class, and then according to the closeness degree of the sample, combine the highest two categories of closeness degree, repeat until all samples are synthesized a class.

Since it is proposed to analyze the taxi resource supply and demand matching degree of different time, from the sky drops and quick smart travel platform, this paper extracts 11 sample locations in Beijing such as Jianguomen, Fuxingmen of taxi distribution and demand in 22 sample time from September 6th to May 7th, 2015. Two ways will be used to answer this question, on the one hand, the taxi resources matching degree of 11 sample sites such as Jianguomen and Fuxingmen in Beijing for cluster analysis; on the other hand, 22 time points from September 6 to 7 in 2015 are chose for cluster analysis to find out the gap of taxi resources matching degree at different time points.

2. The solution of the model

(1) Cluster analysis of samples location

Cluster analysis of sample location has a total of 11 cluster objects, with each object consisting 22 elements (22 time points), and their corresponding elements data can be given by tables.In this problem, the standardized clustering feature is used to make a given data center technology compression process, namely: 


$$
p_{i j}{ }^{*}=\frac{p_{i j}-\bar{p}_{i j}}{s_{i j}}, i=1,2, \cdots, 22, j=1,2, \cdots, 11
$$

The new data obtained with this standardization, the sample mean becomes 0 and variance 1 . Then, the shortest distance method is used to measure the distance between class and class, and create the cluster figure shown in Figure 1.

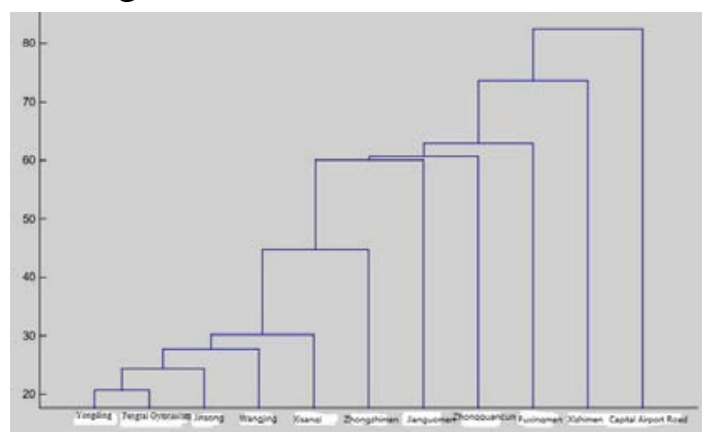

Fig.1 Cluster figure of samples location

There is a big difference in the supply and demand matching situation in various regions. Taxi areas are unevenly distributed.

According to supply and demand matching situation in various regions, the 11 areas are divided into four categories, and the result is:

The first category: Fuxingmen. The second category: Jianguomen, Fuxingmen, Dongzhimen, Xisanqi, Wangjing, Zhongguancun Jinsong, Yongding Gate, Fengtai stadium. The third category: Xizhimen. The fourth category: Capital Airport roads.

According to supply and demand matching situation in various regions, the 11 areas can be divided into five categories, and the result is:

The first category: Zhongguancun. The second category: Jianguomen, Dongzhimen, Xisanqi, Wangjing, Jinsong, Yongding Gate, Fengtai stadium. The third category: Fuxingmen. The fourth category: Xizhimen. The fifth category: capital airport roads.

From the above results combined with the combination distance in clustering figure, it can be seen that the supply and demand matching degree of Fuxingmen, Capital Airport Road, Xizhimen and Zhongguancun is low. The supply and demand matching degree of other places is higher.

(2) Sample time cluster analysis:

By the same token you can get the sample time clustering analysis results shown in Fig.2, which will no longer go into the modeling process.

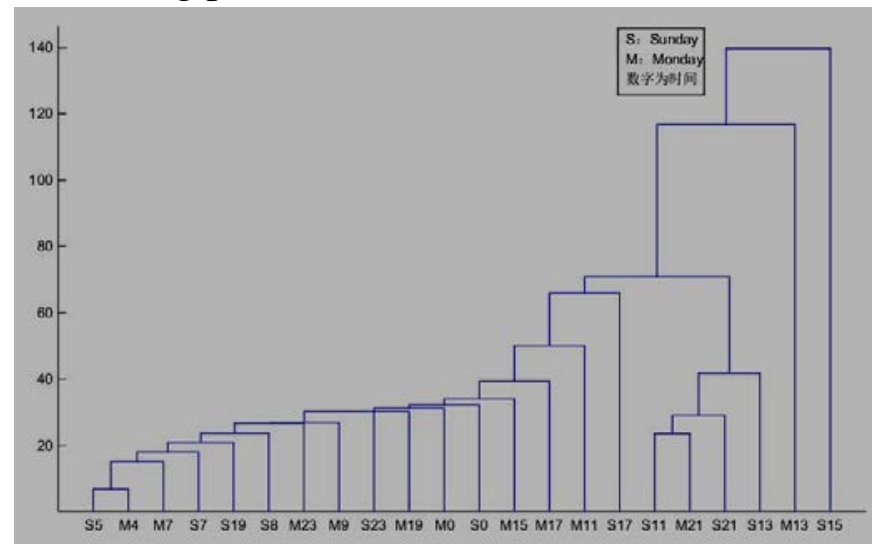

Fig.2 Time sample cluster figure

From the above results combined with the combination distance in clustering figure, it can be seen that the matching degree of taxi resources at different time varies widely. During the working day, the matching degree of to work (7: 00-9: 00) and off work (18: 00-20: 00) period is poor, and at 
other times there is a better matching degree. In the rest day, it is showing poor matching rule. Wherein, the supply and demand matching degree of taxi resources is low in the moments at 11:00, 13:00, 15:00, 17:00, 21:00 on Sunday of September 6 and at 13:00, 21:00 on Monday of September 7 , and that of other time points is higher.

Advantages and disadvantages of the model.Advantages:

(1) The model chooses the taxi supply and taking a taxi demand that directly affects the supply and demand matching degree as an index, which is strong convincing.

(2) The construction of the model is based on a specific, accurate statistics, results are accurate and error is small.

(3) Using cluster analysis for indicators research is scientific and rational, which exactly makes up the deficiencies of lacking classification standard of supply and demand.

Disadvantages:

(1) It doesn't consider the disturbance of traffic congestion and bad weather.

(2) The contrast is not strong, which is unable to further describe the impact of different spatial and temporal factors.

\section{References}

[1] Feng X. Research on taxi development scale at supply and demand equilibrium state. Southwest Jiaotong University, 2010. (In Chinese)

[2] Liu S, Liu N, Zhao Z. Economic analysis of taxi software business model. Gansu Finance, 2014, 06: 68-69. (In Chinese)

[3] He X, Dai Y, Zhou Y. Mathematical Contest in Modeling. Xi'an: Xi'an University Press, 2002. (In Chinese) 